\title{
Crecimiento y producción de Gypsophila paniculata en respuesta al termoperiodo, confinamiento y despunte
}

\section{Growth and production of Gypsophila paniculata in response to thermoperiod, confinement and pinch}

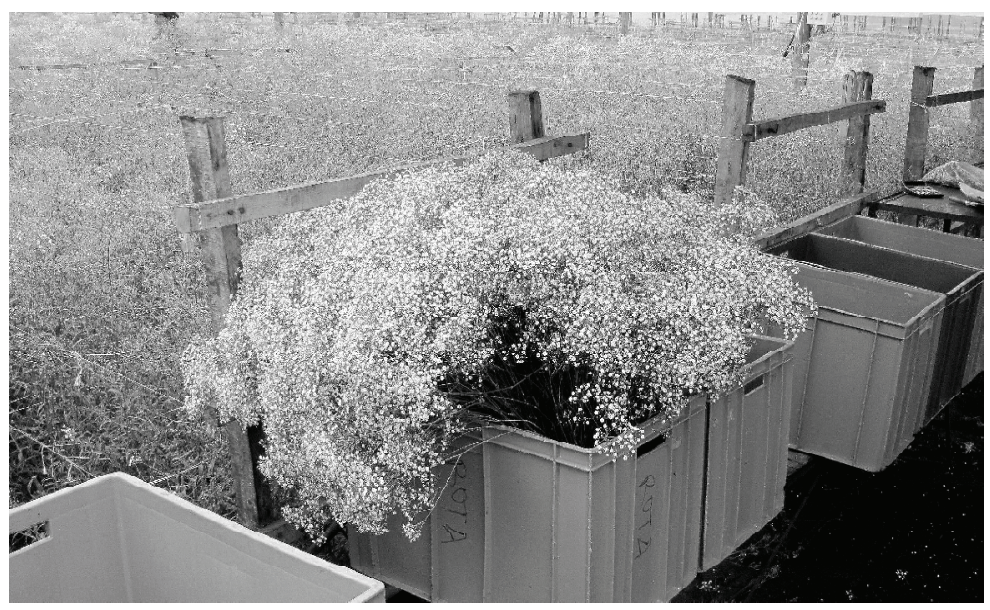

\author{
FÁNOR CASIERRA-POSADA ${ }^{1,3}$ \\ JAIME ERNESTO PEÑA-OLMOS'
}

ANDERSON R. VILLARREAL²

Gypsophila cosechada en una plantación bajo invernadero en la Sabana de Bogotá.

Foto: G. Fischer

\section{RESUMEN}

Gypsophila se utiliza tradicionalmente como acompañante de arreglos y ramos de flores, especialmente de rosas. Las plantas necesitan ciertas condiciones óptimas que promuevan su crecimiento. Una respuesta positiva al termoperiodo y al confinamiento se ha encontrado en muchas plantas. El despunte (pinch) es también un factor importante para determinar el patrón de crecimiento en flores de corte. Se investigó el efecto de temperaturas bajas (vernalización) y del confinamiento sobre el crecimiento, la producción final y la calidad de la flor en plantas de gypsophila sometidas a pinch. El experimento se realizó bajo invernadero en Chía, Cundinamarca. Se utilizaron esquejes de Gypsophila paniculata cv. Perfecta como material vegetal, colocados en suelo como sustrato. Las plantas se arreglaron en un diseño de bloques al azar con ocho tratamientos y cuatro repeticiones. Se evaluó como respuesta el número de brotes viables, el crecimiento y la producción final. Los resultados obtenidos mostraron una influencia positiva del pinch sobre el número de brotes viables, que se incrementaron un 7,84\% en los tratamientos en los cuales se realizó despunte; contrario a las prácticas de confinamiento y vernalización, que no presentaron diferencias significativas para el número de brotes. En cuanto a la altura de la planta, la vernalización aumentó el valor de esta variable 5,96\%, y además redujo el ciclo de cultivo 2,5 semanas con respecto a las plantas control. La mejor producción se presentó cuando las plantas se expusieron al frío, confinamiento y despunte, con un incremento de 5,5\%. En general, la producción total en cuanto al número de ramos cosechados en todos los tratamientos que incluían el confinamiento tuvo un buen comportamiento.

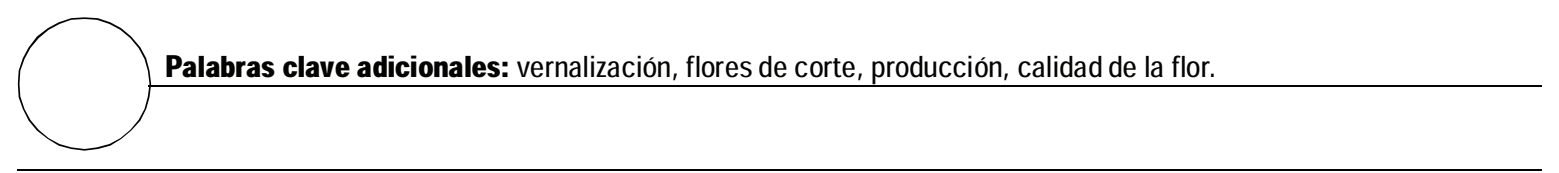

1 Facultad de Ciencias Agropecuarias, Grupo de Investigación Ecofisiología Vegetal, Universidad Pedagógica y Tecnológica de Colombia, Tunja (Colombia).

2 Jefe MIPE, Scarlett's Flowers Ltda., Bogotá (Colombia)

3 Autor para correspondencia: fanor.casierra@uptc.edu.co 


\section{ABSTRACT}

Gypsophila is used traditionally as a filler for formal floral arrangements and bouquets, especially with roses. This plant needs certain optimum conditions that promote its growth. A positive response to thermoperiod and confinement was found in many plants. Pinch is also an important factor determining the growth pattern of cut flowers. The effect of low temperatures (vernalization) and confinement on growth, final yield, and flower quality of pinched gypsophila plants was investigated. The experiment was conducted under greenhouse conditions in Chia, Cundinamarca. Cuttings of Gypsophila paniculata cv. Perfecta were placed in soil. Plants were arranged in a randomized block design with eight treatments and four replicates. The number of viable shoots, growth and final production were evaluated as responses. Results showed a positive influence from pinch on the number of viable shoots, which increased $7.84 \%$ in pinched plants, contrary to the confinement and vernalization treatments, which had no affect on the number of shoots. Vernalization increased the plant height by $5.6 \%$ and reduced the crop cycle by 2.5 weeks, compared to control plants without vernalization. The highest total production was found when plants were exposed to low temperature, confinement and pinch, which increased production by $5.5 \%$. In general, the yield, in terms of harvested flowers, in all treatments for confined plants had a good performance.

Additional key words: vernalization, cut flowers, production, flower quality.<smiles>C1CCCCCC1</smiles>

Colombia cuenta con cerca de 400 empresas dedicadas exclusivamente a la producción de flores de corte con fines de exportación. Aproximadamente existen 8.700 ha dedicadas a la explotación comercial de flores; el $85 \%$ de ellas se ubican en la Sabana de Bogotá; el $12 \%$, en Rionegro y la Ceja, Antioquia, y el $3 \%$, en el Cauca, como Piendamó (Asocolflores, 2010).

La producción y exportación de flores de corte, hace parte de la funcionalidad de la economía, no solo en Colombia, sino en la gran mayoría de países que basan su desarrollo en la agricultura; sin embargo, el pobre desarrollo en estos países ocasiona una disminución en el rendimiento de los cultivos y en el ingreso per cápita de todas las personas dependientes de este tipo de prácticas agrícolas. La Unión Europea es quizás el mayor importador de flores a nivel mundial, con una cifra cercana a los 3.400 millones de euros al año, con lo cual consume aproximadamente el $50 \%$ de la producción mundial (Rosales, 2010). Por otro lado se encuentra Estados Unidos, considerado, al igual que la Unión Europea, un gran consumidor y al mismo tiempo productor de flores de corte a nivel mundial. Los principales proveedores de flores para el mercado norteamericano son Colombia, Ecuador y Costa Rica (Ramos y Merino, 2004).

Entre las flores con mayor demanda y con mayor área sembrada en Colombia se encuentran la rosa y el clavel; adicionalmente, son cultivadas otros flores, Ilamadas rellenos o acompañantes, muy utilizadas para la elaboración de combos y bouquets; entre estas, una de las más cultivadas es Gypsophila paniculata, apreciada principalmente por sus características morfológicas y de calidad. El sector productivo en Colombia considera que, en la actualidad, los cultivos de Gypsophila se encuentran entre el 70 y el $80 \%$ del potencial esperado de producción.

Gypsophila paniculata es una planta herbácea de duración anual, originaria de Europa y Asia occidental, regiones donde predomina el clima templado. Las especies importantes son $G$. elegans y G. paniculata (Matsunaga et al., 1995). De las varias especies del género, solamente $G$. paniculata se ha cultivado comercialmente como flor de corte (Arteaga y Amézquita, 1990). Estas plantas son de día largo, requieren un fotoperiodo de 12 a $18 \mathrm{~h}$ para la iniciación floral, dependiendo del clon. Días largos con temperaturas cálidas 
promueven muy bien la floración. Bajas temperaturas en la noche pueden causar que la planta permanezca en estado vegetativo. Si el promedio de temperaturas mínimas llega a $10^{\circ} \mathrm{C}$, la planta puede tomar $120 \mathrm{~d}$ para florecer, bajo las condiciones australianas (Tregea, 2003); adicionalmente se reporta que temperaturas nocturnas inferiores a $7^{\circ} \mathrm{C}$ favorecen el desarrollo vegetativo, independientemente del número de horas de luz. Con temperaturas medias elevadas durante el cultivo, se acorta el periodo en entrada de producción, todo esto con fotoperiodo favorable, siendo las variedades más precoces las menos exigentes en temperatura (Marín et al., 2006).

La acción vernalizante se lleva a cabo en los meristemos apicales activos (Taiz y Zeiger, 2006). Es por esto que para efectos de una correcta inducción vernalizadora, además de la temperatura y la duración del tratamiento, se debe tener en cuenta el estado de desarrollo de la planta (Roldán y Martínez, 2000). Existen evidencias para creer que la edad de la planta está estrechamente relacionada con la respuesta al termoperiodo; es así como a menor edad de ella, es menor el tiempo requerido para exposición a bajas temperaturas (Peñaloza, 2001).

Los tiempos óptimos para tratamientos vernalizadores en plantas jóvenes se encuentran por debajo de los $10^{\circ} \mathrm{C}$ (Roldán y Martínez, 2000). De igual forma, se cree que el intervalo de temperatura para la inducción y aceleración de la floración se encuentra entre 1 y $7^{\circ} \mathrm{C}$ (Hopkins, 1999). Sin embargo, la eficacia de estos tratamientos con frío para el estímulo de la floración y reducción del ciclo de cultivo está dada por la temperatura constante y sin interrupciones, y por el control de esta en el momento de transplante al sitio definitivo. La desvernalización está dada por interrupciones en el tratamiento frío y por temperaturas por encima de $30^{\circ} \mathrm{C}$ en periodos superiores a $3 \mathrm{~d}$ (Hopkins, 1999; Preece y Read, 1993; Peñaloza, 2001). En plantas de Dianthus alpinus, altas temperaturas durante el periodo de enraizamiento retrasaron el desarrollo y originaron una inducción floral incompleta (Wurr et al., 2000).
El tratamiento vernalizante podría en algún momento desfavorecer el proceso productivo. Es así como en plantas de alcachofa estos tratamientos indujeron un mayor crecimiento en altura (Oyanedel et al., 2004), ocasionando una reducción en la producción final como consecuencia de un crecimiento vegetativo excesivo (Roldán y Martínez, 2000).

Por otro lado, en chile pimiento el despunte temprano desencadenó un mayor rendimiento y no afectó significativamente la calidad de los frutos. Igualmente, el ciclo de cultivo se vio influenciado por el retiro de las yemas terminales, con lo cual se incrementa la productividad anual en función del tiempo (Cruz et al., 2009). De igual manera, en Solanum lycopersicum Mill. se encontró que con la implementación de transplantes tardíos en combinación con despuntes tempranos es posible sostener densidades de siembra elevadas y a la vez reducir el ciclo de cultivo en aproximadamente ocho semanas. EI tiempo de duración de cosecha también se ve afectado por el despunte, concentrándose este en periodos cercanos a $15 \mathrm{~d}$ (Cruz et al., 2009). Lo anterior supone la posibilidad de incrementar la producción de tomate con 3 o 4 ciclos al año, superando el rendimiento potencial de los sistemas convencionales de producción en Europa y Estados Unidos (Sánchez y Ponce, 1998; Sánchez et al., 1999).

Las prácticas de confinamiento son comunes en la aclimatación de plantas multiplicadas in vitro, no obstante, la implementación de estas en la producción hortícola es común, independientemente de la forma de propagación. La obtención de una planta con el sistema radicular bien definido y desarrollado se convierte en el punto de partida para la supervivencia y el crecimiento de esta en el momento del transplante a un sitio definitivo, en el cual encontrará nuevas condiciones ambientales y edafológicas (Bosa et al., 2003). La expansión de las raíces en plantas contenidas en recipientes, sin tener en cuenta el sustrato, varía enormemente en comparación con plantas sembradas directamente en campo (Kämpf, 2000), durante las primeras semanas de crecimiento. En esquejes de G. paniculata var. 'Bristol Fairy', Ia densidad radical se incrementó considerablemente durante el periodo de 
confinamiento, en diferentes sustratos, hasta los 35 d después de iniciada esta etapa (Bosa et al., 2003).

La influencia del termoperiodo y de algunas prácticas de cultivo, como el confinamiento y el despunte, provocan una serie de cambios y adaptaciones tanto en la estructura de la planta como en su rendimiento, ocasionando variaciones en la economía del sector productivo. En la presente investigación se pretendió establecer la importancia de dichas prácticas, así como sus consecuencias en el rendimiento y producción del cultivo de Gypsophila paniculata var. Perfecta, bajo condiciones de invernadero.

\section{MATERIALES Y MÉTODOS}

El ensayo se realizó en el municipio de Chía (Cundinamarca), a $2.600 \mathrm{msnm}$, con temperatura promedio en invernadero de $16{ }^{\circ} \mathrm{C}$, humedad relativa de $85 \%$ y evapotranspiración de 3 mmdía $^{-1}$. Como material vegetal se utilizaron esquejes de Gypsophila paniculata var. Perfecta. Los tratamientos evaluados resultaron de la combinación de tres factores: vernalización (frío), confinamiento y despunte (pinch), para un total de ocho tratamientos: con frío + con confinamiento + sin despunte (testigo); con frío + con confinamiento + con despunte (FCD); con frío + sin confinamiento + sin despunte $(F)$; con frío + sin confinamiento + con despunte (FD); sin frío + con confinamiento + sin despunte (C); sin frío + con confinamiento + con despunte (CD); sin frío + sin confinamiento + sin despunte (O), y sin frío $+\sin$ confinamiento + con despunte (D). Estos tratamientos se agruparon en un diseño de bloques completos al azar, con cuatro repeticiones por tratamiento.

El enraizamiento de los esquejes se realizó en vasos de plástico con capacidad para 3,5 onzas, y se utilizó como sustrato escoria cernida y cascarilla cruda de arroz, con enmiendas de superfosfato triple, magnesio y carbonato de calcio. La vernalización de los esquejes se llevó a cabo durante $56 \mathrm{~d}$ a una temperatura constante de $2^{\circ} \mathrm{C}$, con humedad relativa de $98 \%$, para luego ser ambientados durante $6 \mathrm{~d}$. Posterior al tratamiento frío se realizó el confinamiento de
Ios esquejes en los tratamientos determinados; este consistió en trasladar las plantas a vasos plásticos de 16 onzas de capacidad, utilizando el mismo tipo de sustrato y las mismas condiciones descritas anteriormente. Pasadas tres semanas en zona de confinamiento, las plantas fueron transplantadas a camas definitivas, cada una de ellas con $31,3 \times 0,9 \mathrm{~m}$; antes de la siembra, el suelo se llevó a capacidad de campo, la conductividad eléctrica registrada fue de $2,5 \mathrm{mS}$ $\mathrm{cm}^{-1}$ y el $\mathrm{pH}$ registrado fue de 6,5 , con lo cual no fue necesaria la aplicación de ningún correctivo al suelo. La conductividad eléctrica apropiada para el desarrollo normal de la mayoría de las plantas ornamentales se encuentra entre 2,0 y $3,5 \mathrm{mS} \mathrm{cm}^{-1}$ (Ballester, 1993). De la misma forma, el $\mathrm{pH}$ adecuado para el cultivo de ornamentales se encuentra en el rango de 5,0 a 6,5 (Rodríguez y Medeiros, 2000), y específicamente para $G$. paniculata este puede encontrarse entre 6,5 y 8,0 (Tregea, 2003). La distancia de siembra fue de 40 $\mathrm{cm}$ entre surcos y $30 \mathrm{~cm}$ entre plantas, con una densidad final de $6,5 \mathrm{plantas} / \mathrm{m}^{2}$. Cada tratamiento contó con 80 plantas, para un total de 640 plantas en todo el ensayo.

A partir de los 22 d después de la siembra (dds), todos los tratamientos recibieron 50 noches de luz en sistema de ciclo 8 × 3 ( 8 min de luz y 3 min de oscuridad), el cual se encendía a las 6 p.m. y se apagaba a la 2 a.m. El tratamiento con frío + confinamiento + sin despunte se tomó como testigo, debido a que en el momento de la realización del experimento la finca utilizaba este método para la propagación de los esquejes de G. paniculata.

El despunte se realizó a la quinta semana después de la siembra definitiva en las camas, y se efectuó en el sexto entrenudo para cada planta en los tratamientos con despunte. Todos los tratamientos recibieron aspersiones de ácido giberélico, en concentraciones de $350 \mathrm{ppm}$, a los 22 y 37 dds.

Los datos se recolectaron tomando cuatro plantas por repetición de cada tratamiento, a las cuales se les llevaron registros de crecimiento semanalmente, y de número de brotes viables para cosecha (aquellos que tuvieran buenas condiciones como forma y tamaño) a la décima semana, en la cual fueron evidentes las 
diferencias con respecto a brotes inviables. El experimento tuvo una duración de 21 semanas, y al final del ciclo productivo se llevó el registro diario de producción de ramos GSP, que son aquellos que tienen como peso mínimo $280 \mathrm{~g}$ y su elaboración se puede hacer con 4 a 18 varas, y de ramos GY10, que son los que tienen 10 varas únicamente, sin importar su peso, y que se elaboran con todas las varas de menor consistencia y peso.

El manejo del riego, la fertilización y el control de plagas y enfermedades se realizó bajo esquemas preestablecidos y direccionados por el departamento técnico y el departamento MIPEMIRFE de la finca en la cual se realizó el estudio.

EI análisis estadístico se realizó mediante la versión 11.5.1 de la aplicación SPSS (Statistical Product and Service Solutions, Chicago, IL).

\section{RESULTADOS Y DISCUSIÓN}

\section{Número de brotes viables}

La influencia del frío, o el confinamiento sobre el número de brotes viables en gypsophila, fue nula, debido a que no se registraron diferencias significativas entre las plantas con y sin estos factores. Por el contrario, los días hasta la brotación en peonia (Paeonia sp.), cultivares Coral Sunset (CS), Monsieur Jules Elie (MJE) y Sarah Bernhardt (SB) disminuyeron a medida que se incrementó la duración del tratamiento frío y decreció la temperatura de refrigeración (Fulton et al., 2001). De igual forma, el número de brotes en peonia aumentó considerablemente con el tratamiento de frío por 12 semanas con respecto al de 3 semanas (Fulton et al., 2001). Lo anterior sugiere que la duración del tratamiento frío está directamente relacionada con la brotación en algunas especies (Andrés y Durán, 1999; Citadin et al., 2001; Warmund y Krumme, 2005).

Por el contrario, el despunte indujo un mayor número de brotes viables a la décima semana después de la siembra, aumentando el 7,84\% el valor de esta variable en las plantas a las cuales se retiró el ápice. De la misma forma, las plantas de los tratamientos con despunte mostraron una brotación más uniforme y ordenada que las que no fueron despuntadas. Este efecto se debe a que si el ápice es eliminado, los meristemos axilares más cercanos toman su rol, ocasionando una brotación más uniforme de ramas laterales (figura1).

\section{Crecimiento y ciclo de cultivo}

La vernalización ejerció una influencia positiva sobre el alargamiento del tallo en las plantas de gypsophila ; así, las que recibieron tratamiento frío fueron $5,96 \%$ más altas que aquellas que no lo recibieron, en estas últimas también se evidenció el alargamiento del ciclo de cultivo en, aproximadamente, 2,5 semanas. Por otro lado, se observó que después de la quinta semana el crecimiento mostró una aceleración en todas las plantas; sin embargo, la observación permitió establecer que aquellas plantas que recibieron el despunte tuvieron un crecimiento homogéneo en cada uno de sus tallos, a diferencia de las plantas sin despunte, en las cuales el tallo central mostró un mejor desarrollo respecto a las demás (figura 2).

Se observaron cambios en el crecimiento de las plantas con y sin vernalización, de tal manera que las primeras tuvieron un incremento significativo en la altura de la plantas a partir de la séptima semana (49 dds), y las segundas demoraron entre una y dos semanas más para iniciar esta etapa de desarrollo (entre 56 y 63 dds). El crecimiento en altura finalmente se detiene en la semana 12 (84 dds) para las plantas sometidas a vernalización, y se alarga entre 2 y 4 semanas (entre 98 y 112 dds) para las plantas sin tratamiento con frío. A partir de este momento la planta comienza la etapa de llenado y apertura de las primeras flores. La cosecha en las plantas bajo tratamiento frío inició entre 2 y 4 semanas antes que las plantas sin vernalización.

El crecimiento se presentó para todos los tratamientos como una curva sigmoide en la cual se distinguieron tres fases; la fase logarítmica, la fase exponencial o lineal y por último la fase de senectud. Para los tratamientos que recibieron vernalización, la fase logarítmica se presentó de la semana cero a la semana cuatro, en donde las plantas iniciaron su proceso de establecimiento. En los tratamientos que no recibieron vernalización dicha fase se presentó de la semana 


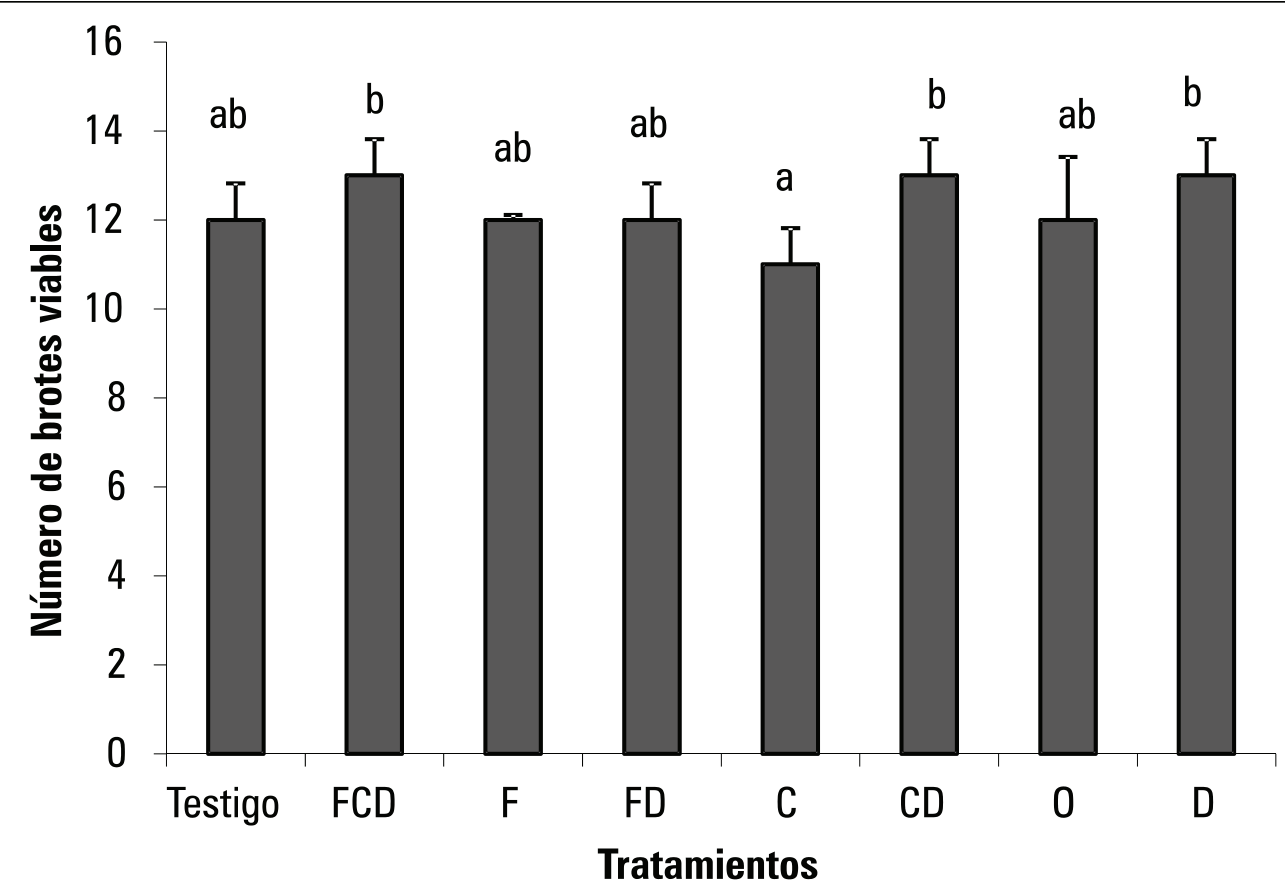

Figura 1. Número de brotes viables por tratamiento en plantas de Gypsophila paniculata var. Perfecta sometidas a termoperiodo, confinamiento y despunte, 70 dds. Testigo: con frío + con confinamiento + sin despunte; FCD: con frío + con confinamiento + con despunte; F: con frío + sin confinamiento + sin despunte; FD: con frío + sin confinamiento + con despunte; $C$ : $\sin$ frío + con confinamiento + sin despunte; CD: sin frío + con confinamiento + con despunte; 0 : sin frío + sin confinamiento + sin despunte; D: sin frío + sin confinamiento + con despunte. Promedios con letras distintas indican diferencia significativa según la prueba de Tukey $(P<0,05)$.

cero a la semana seis. Fue en la fase exponencial o lineal que el crecimiento aumentó en forma constante, a medida que la fotosíntesis actuó sobre la planta, incrementándose también el volumen y el peso. Para los tratamientos que recibieron frío, esta fase se desarrolló entre la semana cinco y la semana doce, mientras que para los tratamiento sin frío ocurrió de la semana siete a la semana quince después de la siembra; enseguida, para ambos grupos de tratamientos, comenzó la fase de senectud.

Estas fluctuaciones en el crecimiento de los vegetales son influenciadas directamente por factores como la temperatura y la luz; sin embargo, en este ensayo el factor luz fue constante para todos los tratamientos, mientras que la temperatura no lo fue. En Dianthus sp. se reportan requerimientos de vernalización durante la etapa temprana de crecimiento, lo cual induce el desarrollo de la planta $y$, consecuentemente, la apertura de la flor (NappZinn, 1987). Existen variaciones en el tiempo de floración de esquejes de Dianthus allwoodii y $D$. alpinus, inducidas por bajas temperaturas en la planta madre antes de separar los esquejes. No obstante, la variación en la floración de esquejes de Dianthus allwoodii se vio favorecida únicamente por temperaturas bajas, mientras que los esquejes de $D$. alpinus florecieron con temperaturas bajas, seguidas de variaciones subsecuentes en las mismas, que teóricamente no son aptas para la inducción de la floración (Wurr et al., 2000). Del mismo modo, el estímulo vernalizante puede ser transmitido a través de cortes en Morifolium crisantemo (Schwabe, 1954) y en $D$. caryophyllus en condiciones de invernadero (Bunt y Cockshull, 1985).

En el presente ensayo, las variaciones en el termoperiodo indujeron determinados cambios en la fisiología de la planta, los cuales, para el caso de las plantas con vernalización, aceleraron el desarrollo y la floración, disminuyendo el ciclo de cultivo y, de esta manera, los costos de producción. Se ha encontrado que Gypsophila es 


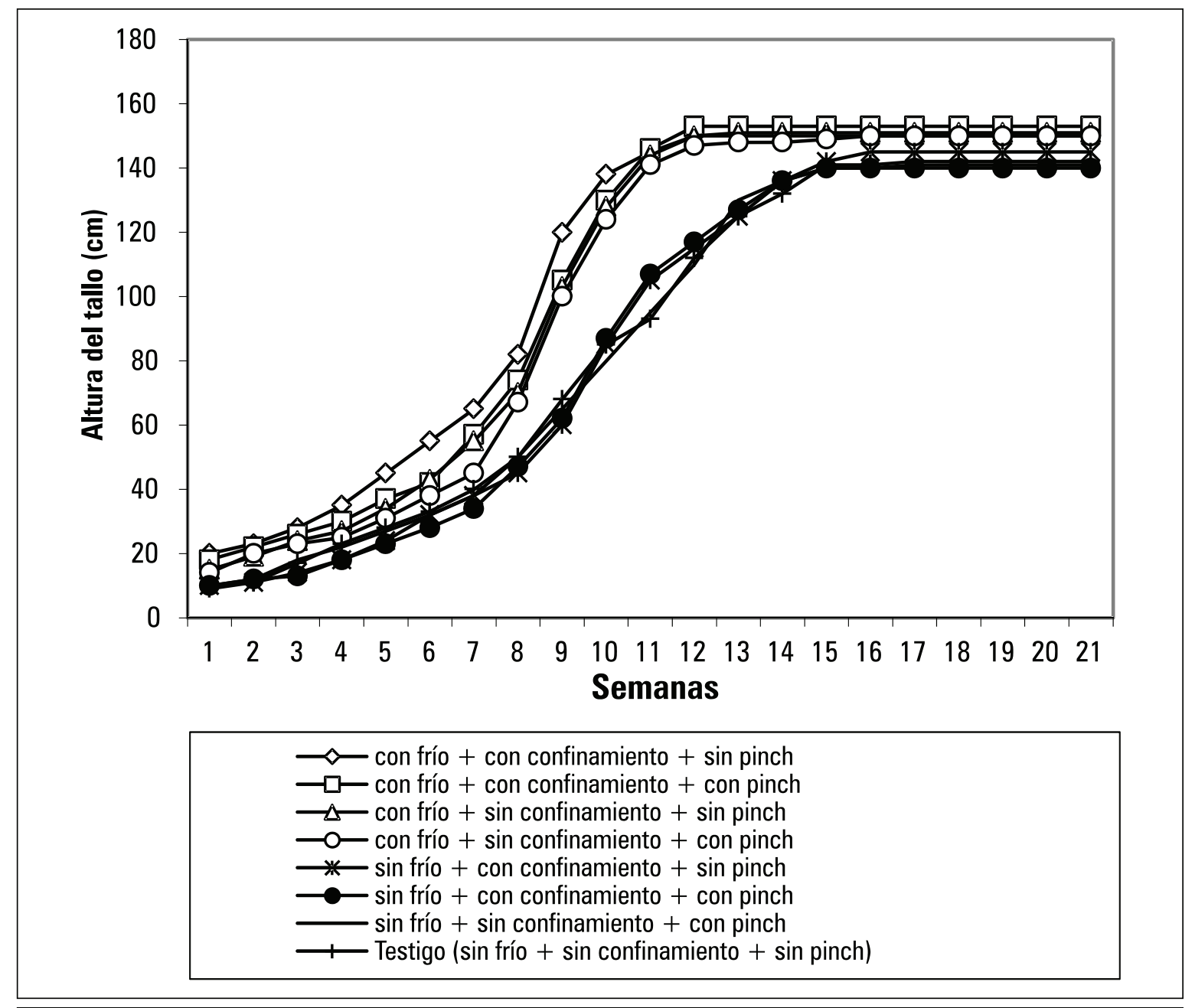

Figura 2. Curvas de crecimiento en plantas de Gypsophila paniculata var. Perfecta sometidas a termoperiodo, confinamiento y despunte.

una planta absolutamente de día largo, con respuesta cuantitativa a la vernalización. Los métodos desarrollados para controlar su floración incluyen el almacenamiento en frío de plántulas enraizadas antes de la siembra e iluminación nocturna complementaria. Es así como el desarrollo y la inducción floral están directamente relacionados con la temperatura (Halevy, 1999). La vernalización acelera la inducción de la floración, promueve la elongación de los tallos y acorta el ciclo del cultivo, mientras que el pinch promueve un aumento en la brotación y producción de tallos en gypsophila, pero alarga el ciclo de cultivo (León, 2004). Lo anterior podría contrastar con lo encontrado en el presente ensayo, ya que la duración del ciclo para las plantas sometidas a los tratamientos sin frío + sin confinamiento + sin despunte y sin frío + sin confinamiento + con despunte fue la misma. Se podría inferir, entonces, que el pinch no alarga el ciclo de cultivo, pero tampoco tiene un efecto sinérgico con los tratamientos de frío en plantas de gypsophila. De forma contraria, sí se detectó el sinergismo de la combinación frío + confinamiento, para la iniciación de la floración en plantas de G. paniculata.

El ciclo de cultivo de las diferentes especies ha sido ampliamente estudiado. Las variaciones con respecto al termoperiodo parecen ser el punto de estudio más importante. En cultivos de chile pimiento fue posible disminuir el ciclo de cultivo en 6,5 semanas, aproximadamente, por medio de la implementación de prácticas como el despunte 
temprano y elevadas densidades de siembra, lo cual permitió producir de 2,3 a 3,2 ciclos por año (Cruz et al., 2009).

La distancia de siembra óptima para el desarrollo de gypsophila es muy variada; se encuentra alrededor de $30 \mathrm{~cm}$ entre plantas y entre 50 y 80 $\mathrm{cm}$ entre hileras (Tregea, 2003). Otros autores afirman que la densidad de siembra está alrededor de 5 plantas $/ \mathrm{m}^{2}$, con distancias de siembra entre hileras de 40 a $50 \mathrm{~cm}$ y entre plantas de $40 \mathrm{~cm}$ (Marín et al., 2006). Lo anterior contrasta con lo realizado en el presente ensayo, en el cual se utilizó una distancia de siembra de $40 \mathrm{~cm}$ entre surcos y de $30 \mathrm{~cm}$ entre plantas. Probablemente, el efecto del despunte y la vernalización estuvo relacionado con la reducción del ciclo de cultivo en el presente experimento, lo cual, sumado la densidad de siembra, se hizo más evidente. No obstante, en cultivos de Gypsophila se reportó un aumento del ciclo de cultivo al someter los esquejes a tratamiento con frío y despunte, en comparación con la aplicación de frío sin despunte (León, 2004). Lo anterior, supone la importancia de la vernalización para reducir los ciclos de cosecha en el cultivo de gypsophila, independientemente de la densidad y el pinch.

No se debe olvidar que el rendimiento de la planta puede ser afectado negativamente por la densidad de población, y que, por el contrario, el rendimiento por unidad de superficie se puede incrementar. La disminución del rendimiento de la planta con altas densidades de siembra es atribuida a una menor intercepción de la radiación por parte del vegetal (Batal y Smittle, 1981; Stoffella y Bryan, 1988; Decoteau y Graham, 1994; Cebula, 1995; Lorenzo y Castilla, 1995), sin embargo, es posible que el despunte temprano haya disminuido esta situación en las plantas de Gypsophila. Plantas sembradas a alta densidad y con despunte temprano logran distribuir mejor la radiación fotosintéticamente activa, con lo cual alcanzan de forma mucho más rápida un índice de área foliar óptimo (Cruz et al., 2009).

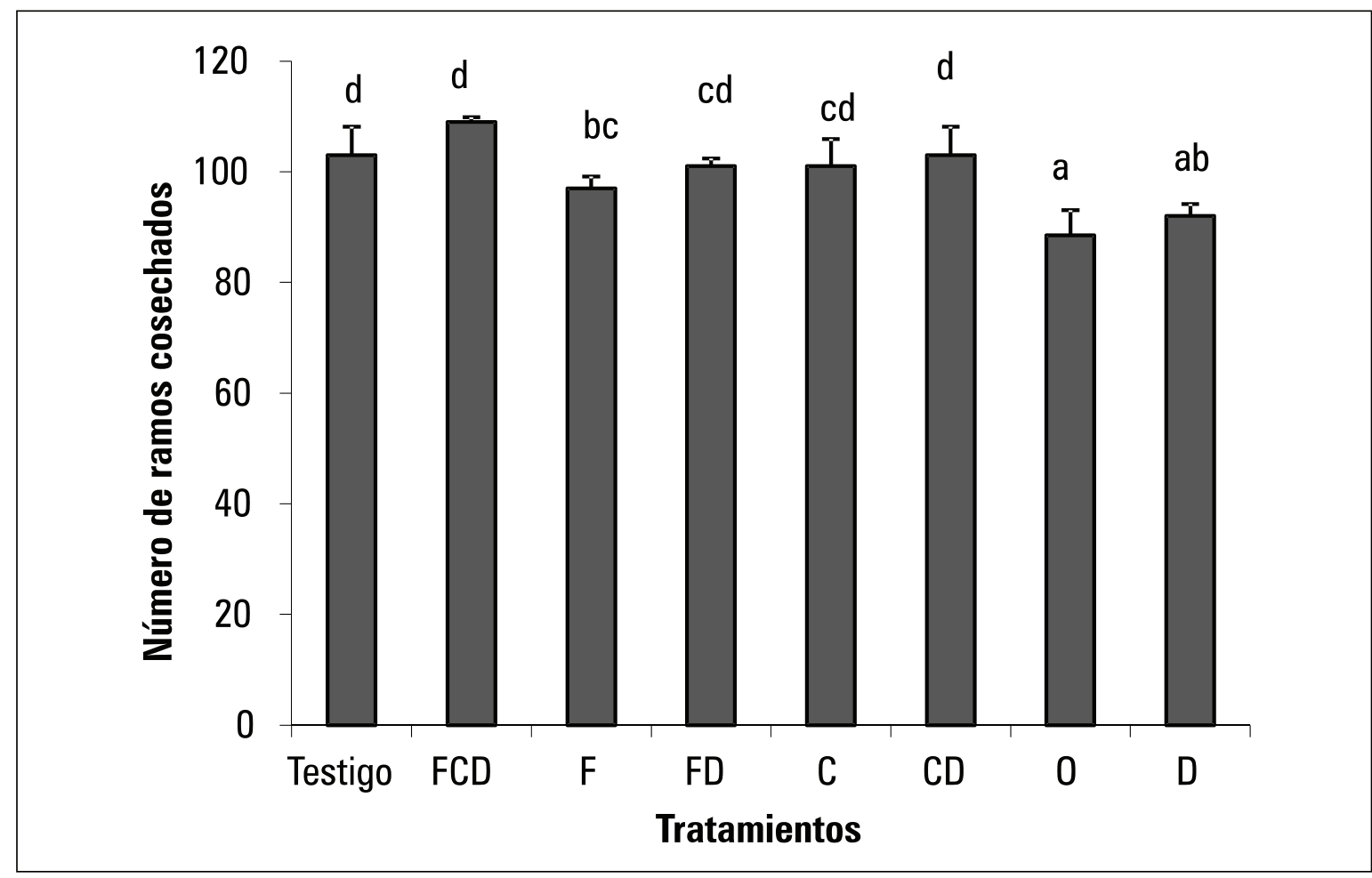

Figura 3. Número de ramos cosechados a los 147 dds en plantas Gypsophila paniculata var. Perfecta sometidas a termoperiodo, confinamiento y despunte. Testigo: con frío + con confinamiento + sin despunte; FCD: con frío + con confinamiento + con despunte; F: con frío + sin confinamiento + sin despunte; FD: con frío + sin confinamiento + con despunte; C: $\sin$ frío + con confinamiento + sin despunte; CD: sin frío + con confinamiento + con despunte; 0: sin frío + sin confinamiento + sin despunte; $D:$ sin frío + sin confinamiento + con despunte. Promedios con letras distintas indican diferencia significativa según la prueba de Tukey $(P<0,05)$. 


\section{Producción final}

La producción final se vio influenciada por la interacción entre los factores en estudio. Es así como los tratamientos testigo, con frío + con confinamiento + con despunte y sin frío + con confinamiento + con despunte, presentaron diferencia significativa, en comparación con los tratamientos con frío + sin confinamiento + sin despunte, sin frío + sin confinamiento $+\sin$ despunte y sin frío + sin confinamiento + con despunte. Sin embargo, los tres primeros no presentaron diferencia significativa con respecto al tratamiento sin frío + con confinamiento + sin despunte, con lo cual se establece que el confinamiento, definitivamente, favorece la producción.

La combinación de los tres factores en el tratamiento con frío + con confinamiento + con despunte incrementó la producción un 5,05\% con respecto al testigo, y a pesar de que las diferencias no fueron significativas, es importante lograr un aumento productivo, teniendo en cuenta que el potencial esperado por los productores dista de la producción actual. La importancia de cada uno de estos factores se reflejó en el resultado del tratamiento sin frío + sin confinamiento + sin despunte, en el cual la producción se redujo 18,8\%. Al respecto, Gudin (1992) encontró que los esquejes de rosa (Rosa hybrida, cv. Meijikatar) sometidos a vernalización durante un mes después del corte, y plantados en invernadero, presentaron un incremento en la producción. Por tanto, cualquier práctica de manejo de cultivo que se encuentre dentro de los parámetros rentables y contribuya al aumento de la producción se convierte en alternativa para la tecnificación y mejoramiento de esta en cultivos de flores como Gypsophila paniculata.
La eficiencia productiva de un cultivo de flores está expresada principalmente por el mayor número de flores formadas, siendo esta la variable final más importante del rendimiento. Estadísticamente se confirmó que el tratamiento con frío es una de las variables más importantes en el aumento de la producción de flores, y que el confinamiento conduce a un mejor desarrollo radicular de las plantas, las cuales inician de esta manera el proceso de establecimiento en campo mucho más rápido, de tal forma que la reducción del ciclo de cultivo también se hizo evidente en las plantas confinadas, con excepción del tratamiento sin frío + con confinamiento + con despunte, el cual no tuvo vernalización. En plantas de peonia la producción aumentó a medida que se incrementó el tiempo de vernalización. Sin embargo, en la misma especie, el cultivar Coral Sunset no mostró aumento en la producción de flores con respecto al tiempo de refrigeración (Fulton et al., 2001).

\section{Producción de ramos GSP y GY10}

La producción de los tratamientos fue divida según la clase de ramos cosechados, con las especificaciones técnicas de corte para Gypsophila paniculata var. Perfecta, en la finca en la cual se realizó el estudio. Todos los tratamientos que recibieron despunte incrementaron la producción de ramos GSP, lo cual, económicamente, aumenta la rentabilidad del cultivo, debido a la calidad de los tallos en estos ramos. Al realizar el despunte, la planta distribuye mejor la brotación de varas $y$, además, se induce el engrosamiento de estas. 


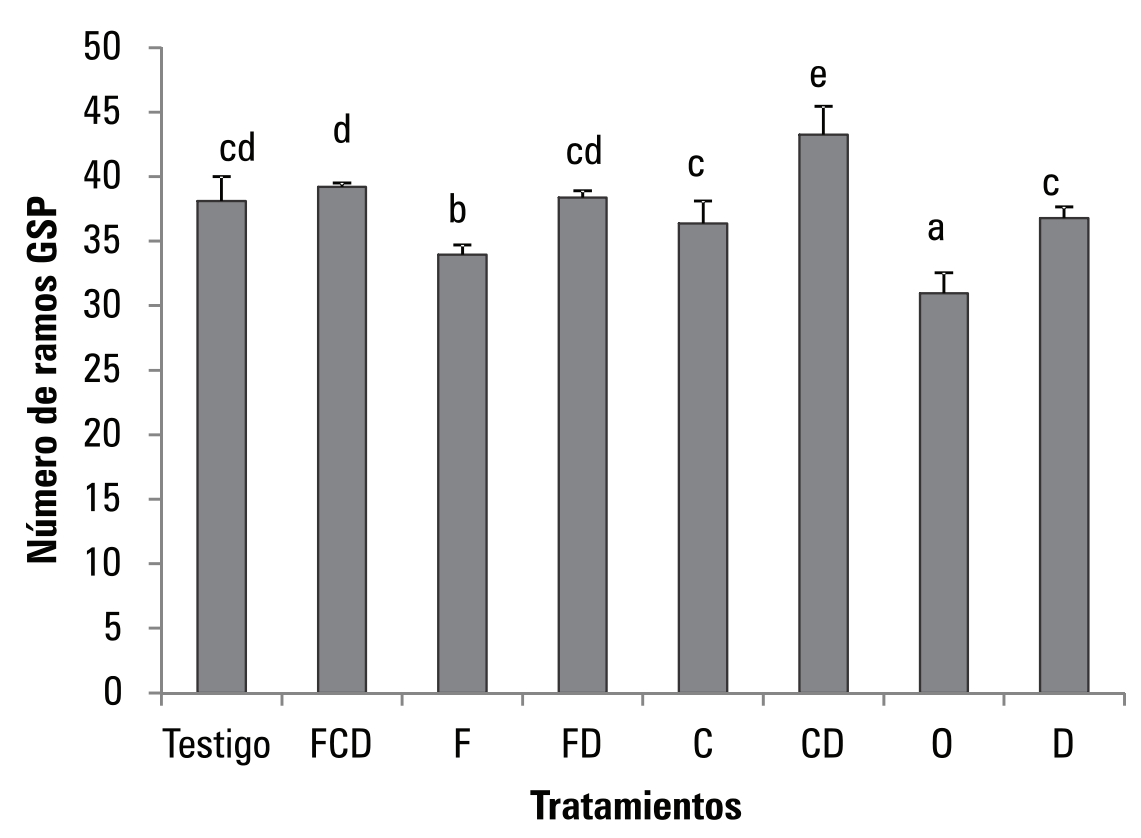

Figura 4. Número de ramos GSP en plantas Gypsophila paniculata var. Perfecta sometidas a termoperiodo, confinamiento y despunte, a los 147 dds. Testigo: con frío + con confinamiento + sin despunte; FCD: con frío + con confinamiento + con despunte; F: con frío + sin confinamiento + sin despunte; FD: con frío + sin confinamiento + con despunte; C: sin frío + con confinamiento + sin despunte; CD: sin frío + con confinamiento + con despunte; 0 : sin frío + sin confinamiento + sin despunte; D: sin frío + sin confinamiento + con despunte. Promedios con letras distintas indican diferencia significativa según la prueba de Tukey $(P<0,05)$.

La comercialización de los ramos GSP genera mayor rentabilidad que la de los GY10, por esta razón, y sin desconocer que ambos tipos de ramos son comercializables, es deseable que en la producción de gypsophila el número de ramos GSP aumente y disminuya el número de ramos GY10. Igualmente, los tratamientos confinados presentaron un aumento en la producción de ramos GSP, con lo cual se evidencia sinergismo en la combinación de estos dos factores. Las plantas sometidas al tratamiento sin frío + con confinamiento + con despunte presentaron el mayor valor de ramos GSP, aumentando 11,9\% con respecto al testigo y $9,34 \%$ con respecto al tratamiento con frío + con confinamiento + con despunte. Sin embargo, es posible que la ausencia de vernalización induzca una mayor producción de ramos GSP, pero alargue el ciclo de cultivo aproximadamente tres semanas, lo cual no resultaría económicamente rentable.

El incremento en el crecimiento, desarrollo y producción de varias especies vegetales parece estar directamente relacionado con un buen desarrollo radicular (Bosa et al., 2003), con aspectos de manejo de cultivo como el pinch (León, 2004; Cruz et al., 2009) y con la duración del tratamiento frío (Fulton et al., 2001). La luz, la nutrición (Powell, 1987), la temperatura (Jacobs et al., 2002) y otros factores ambientales pueden cambiar las necesidades de temperatura de la planta en función del tiempo (Rahemi y Pakish, 2009).

Con base en la información obtenida se puede concluir que el crecimiento de G. perfecta se ve directamente afectado por el tratamiento con frío (vernalización) en condiciones tropicales, para lo cual se hace necesario someter las plantas a temperaturas bajas $\left(2^{\circ} \mathrm{C}\right)$, con el propósito de tener un mejor crecimiento de ellas y garantizar que la producción no se retrase de manera significativa. Adicionalmente, el frío y el confinamiento hacen que la producción de ramos sea óptima, aunque cabe destacar que los tratamientos que no recibieron frío, pero fueron confinados, tuvieron buenos rendimientos, lo que indica que si no se someten las plantas al frío, lo ideal sería someterlas a confinamiento. 


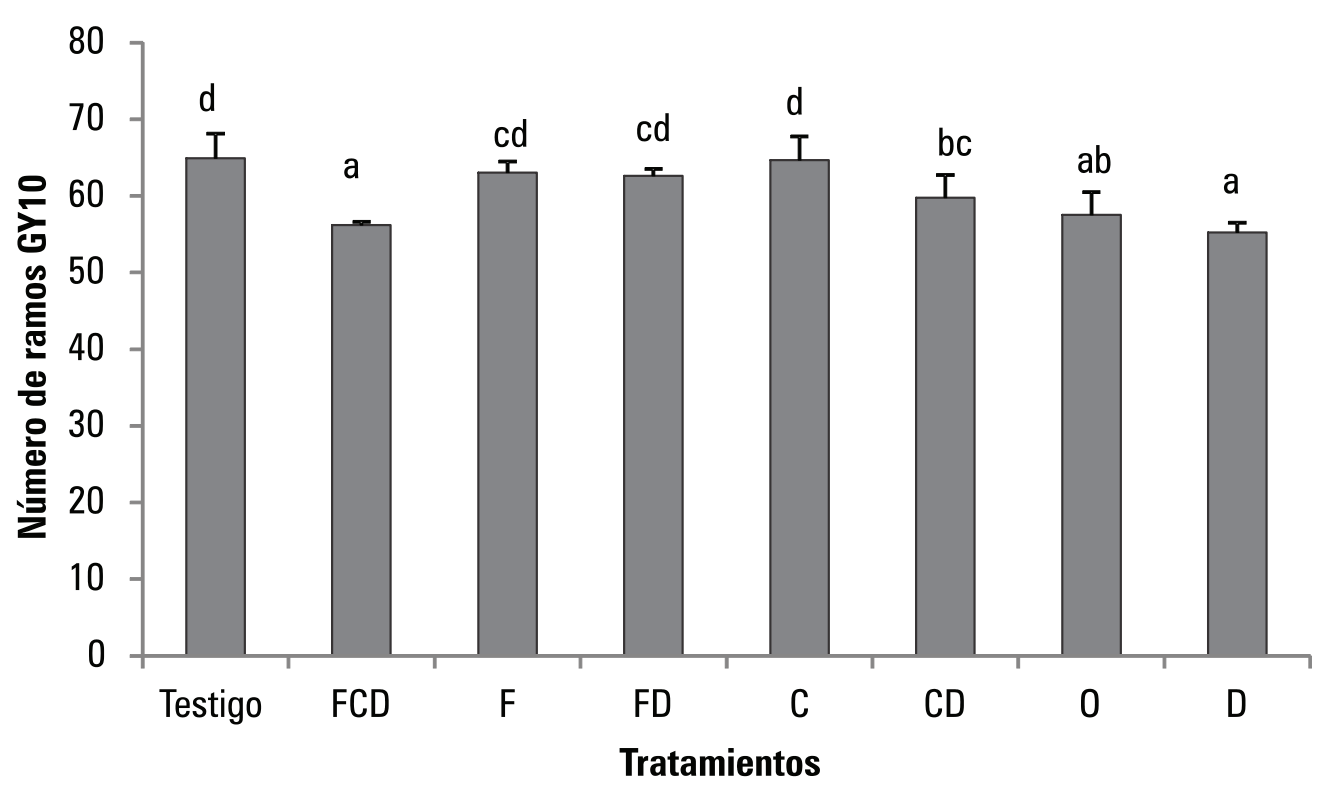

Figura 5. Número de ramos GY10 en plantas Gypsophila paniculata var. Perfecta sometidas a temoperiodo, confinamiento y despunte, a los 147 dds. Testigo: con frío + con confinamiento + sin despunte; FCD: con frío + con confinamiento + con despunte; F: con frío + sin confinamiento + sin despunte; FD: con frío + sin confinamiento + con despunte; C: sin frío + con confinamiento + sin despunte; CD: $\sin$ frío + con confinamiento + con despunte; $0: \sin$ frío + sin confinamiento + sin despunte; D: sin frío + sin confinamiento + con despunte. Promedios con letras distintas indican diferencia significativa según la prueba de Tukey $(P<0,05)$.

La ausencia de frío, al combinarse con el confinamiento y el despunte, resulta ser un procedimiento que induce en las plantas buen desarrollo y productividad. Teniendo en cuenta el ahorro de tiempo y el costo del tiramiento con frío, el confinamiento y el despunte pueden llegar a compensarlo, sin embargo, se requieren los análisis de costos y rentabilidad en cada empresa en particular.

Todos los tratamientos que no recibieron frío alargaron su ciclo de cultivo entre 14 y $28 \mathrm{~d}$, lo cual resulta contraproducente, puesto que la producción de flores de corte para exportación requiere en lo posible el mayor número de ciclos al año, en aras de alcanzar una alta producción.
El despunte de varas dominantes en gypsophila mejora la brotación de ramas laterales de todos los tratamientos, por efecto de eliminación de la dominancia apical y la redistribución de las concertaciones de reguladores del crecimiento.

El mejor coeficiente de productividad asociado al menor ciclo de cultivo se obtuvo con el tratamiento que recibió frío, confinamiento y despunte; de igual manera, este tratamiento fue el que mayor número de ramos GSP produjo. Sin embargo, es importante anotar que las plantas que no se sometieron a frío, expuestas a confinamiento y con despunte, presentaron el mejor porcentaje de ramos GSP.

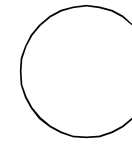

AGRADECIMIENTOS

Este estudio fue desarrollado con el apoyo de la Dirección de Investigaciones (DIN) de la Universidad Pedagógica y Tecnológica de Colombia, Tunja, en el marco del plan de trabajo del Grupo de Investigación Ecofisiología Vegetal, adscrito al programa de Ingeniería Agronómica de la Facultad de Ciencias Agropecuarias. 


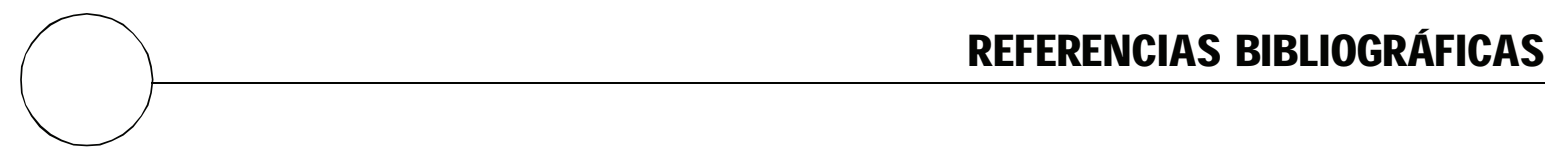

Andrés, M.V. y J.M. Durán. 1999. Cold and heat requirement of apricot tree (Prunus armeniaca L.). J. Hort. Sci. Biotechnol. 74, 757-761.

Arteaga, A.D. y M.O. Amézquita. 1990. Efecto de la zona de localización del esqueje en la planta madre sobre el enraizamiento de Gypsophila paniculata L. Agron. Colomb. 47, 4-53.

Asociación Colombina de Exportadores de Flores. 2010. Aspectos de producción. En: http:// www.asocolflores.org/; consulta: septiembre de 2010.

Ballester, O.J.F. 1993. Substratos para el cultivo de plantas ornamentales. Valencia, España.

Batal, K.M. y D.A. Smittle. 1981. Response of bell pepper to irrigation, nitrogen, and plantpopulation. J. Amer. Soc. Hort. Sci. 106, 259262.

Bosa, N.; E.O. Calvete; V.A. Klein y M. Suzin. 2003. Crescimento de mudas de gipsofila em diferentes substratos. Hortic. Bras. 21(3), 514-519.

Bunt, A.C. y K.E. Cockshull. 1985. Dianthus caryophyllus. pp. 430-432. En: Halevy, A.H. (ed.). CRC Handbook of Flowering, Vol. II. CRC Press, Boca Raton, FL.

Cebula, S. 1995. Optimization of plant and shoot spacing in greenhouse production of sweet pepper. Acta Hort. 412, 321-329.

Citadin I.; M.C. Raseira; F.G. Herter y J.B. Silva. 2001. Heat requirement for blooming and leafing in peach. HortScience 36, 305-307.

Decoteau, D.R. y H.A.H. Graham. 1994. Plant spatial arrangement affects growth, yield, and pod distribution of cayenne peppers. HortScience. 29, 149-151.
Cruz, H.N.; F. Sánchez; J. Ortiz y M. Mendoza. 2009. Altas densidades con despunte temprano en rendimiento y período de cosecha en chile pimiento. Agricultura Técnica en México 35(1), 70-77.

Fulton, T.A.; A.J. Hall y J.L. Catley. 2001. Chilling requirements of Paeonia cultivars. Sci. Hortic. 89, 237-248.

Gudin, S. 1992. Influence of bud chilling on subsequent reproductive fertility in roses. Sci. Hortic. 51(1-2), 139-144.

Halevy, A.H. 1999. Ornamentals: where diversity is king - the israeli experience. pp. 404-406. En: Janick, J. (ed.). Perspectives on new crops and new uses. ASHS Press, Alexandria, VA.

Hopkins, W. 1999. Introduction to plant physiology. $2^{\text {nd }}$ ed. Courier Companies Inc., New York, NY.

Jacobs, J.N.; G. Jacobs y N.C. Cook. 2002. Chilling period influences the progression of bud dormancy more than does chilling temperature in apple and pear shoots. J. Hort. Sci. Biotechnol. 77, 333-339.

Kämpf, A. 2000. Produção comercial de plantas ornamentais. Agropecuária, Guaíba (RS), Brasil.

Lorenzo, P. y N. Castilla. 1995. Bell pepper yield response to plant density and radiation in unheated plastic greenhouse. Acta Hort. 412, 330-334.

León, R. S. 2004. Efectos del manejo de pinch en la producción de gypsophila (Gypsophila paniculata $L$.), variedad Perfecta con siembra invernada y sin invernar, bajo cubierta, en Quiroga - provincia de Imbabura. En: http:/ /www.pucei.edu.ec:1640/publicaciones/ index.php/tesis-de-grado/tesis-de-grado/ 
doc_details/10-efectos-del-manejo-de-pinch-enla-produccion-de-gypsophila-gypsophilapaniculata-I.html; consulta: septiembre de 2010.

Matsunaga, M.; S. T. Arruda; A. D. Almeida Jr. y M.P. Almeida. 1995. Custo e rentabilidade na produção de Gypsophila, região de Atibaia, estado de São Paulo, 1994. Informações Econômicas 25(10), 69-76.

Marín, J. L.; M. R. González; A. G. Benavente G. y L.G. Abellón. 2006. Complementos ornamentales de verde y flor. Serie Técnica y de Estudios 30. En: http://www.fyta.es/ images/flores.pdf; consulta: septiembre de 2010.

Napp-Zinn, K. 1987. Vernalization environmental and genetic regulation. pp. 123-132. En: Atherton, J.G. (ed.). Manipulation of flowering. Butterworths, London.

Oyanedel, M.E.; P. Peñaloza A. y L. Rojo V. 2004. Desarrollo de un sistema de producción forzada de alcachofa mediante vernalización artificial y GA3. En: Pontificia Universidad Católica de Valparaíso. Disponible en: http:/ /ucv.altavoz.net/prontus_unidacad/site/ artic/20061214/asocfile/20061214154319/ rojo_Iorena.pdf; consulta: septiembre de 2010.

Peñaloza, P. 2001. Semillas de hortalizas: Manual de producción. Ediciones Universitarias de Valparaíso, Valparaíso, Chile.

Powell, L.E. 1987. Hormonal aspects of bud and seed dormancy in temperate-zone woody plants. HortScience 22, 845-850.

Preece, E. y E. Read. 1993. The biology of horticulture. An introductory textbook. John Wiley Sons Inc., New York, NY.

Rahemi, M. y Z. Pakkish. 2009. Determination of chilling and heat requirements of pistachio (Pistacia vera L.) cultivars. Agric. Sci. China 8(7), 803-807.

Ramos, M.J. y M. Merino P. 2004. Mercado internacional de flor y verdes de corte. Horticultura 181, 32-36.

Rodríguez, L.T. y C.A.B. Medeiros. 2000. Caracterização química de substratos constituídos de diferentes misturas de turfa com casca de acácia e casca de arroz carbonizada. pp. 50-51. En: Resumos Encontro Nacional sobre Substratos para Plantas.. UFSC, Florianópolis, Brasil.

Roldán, M. y J. Martínez. 2000. Floración y su control ambiental. pp. 403-417. En: AzcónBieto, J. y M. Talón (eds.). Fundamentos de fisiología vegetal. McGraw-Hill, Barcelona, España.;

Rosales, G. 2010. Algunos aspectos del comercio mundial de las flores. En: http:// untraflores.org/index.php?option $=$ com_content\&task =view\&id $=296 \&$ Itemid $=1$; consulta: septiembre de 2010.

Sánchez, del C.F.; C.J. Ortiz; C.M.C. Mendoza; H.V.A. González y L.M.T. Colinas. 1999. Características morfológicas asociadas con un arquetipo de tomate apto para un ambiente no restrictivo. Agrociencia 33, 2129.

Sánchez, del C.F. y O.J. Ponce. 1998. Densidades de población y niveles de despunte en tomate (Solanum lycopersicum Mill.) cultivado en hidroponía. Revista Chapingo, Serie Horticultura 4, 89-94.

Schwabe, W.W. 1954. Factors controlling flowering in the chrysanthemum. IV. The site of vernalization and translocation of the stimulus. J. Exp. Bot. 5, 389-400.

Stoffella, P.J. y H.H. Bryan. 1988. Plant population influences growth and yields of bell pepper. J. Amer. Soc. Hort. Sci. 113, 835 839. 
Taiz, L. y E. Zeiger. 2006. Fisiología vegetal. 3a. ed. Publicaciones de la Universitat Jaume I. Castellón, Valencia, España.

Tregea, W. 2003. Gypsophila growing in central Australia. En: http://www.nt.gov.au/d/ Content/File/p/Garden/615.pdf; consulta: septiembre de 2010 .
Warmund, M.R. y J. Krumme. 2005. A chilling model to estimate rest completion of erect blackberries. HortScience 40, 1259-1262.

Wurr, D.C.E.; J.R. Fellows y L. Andrews. 2000. The effects of temperature and daylength on flower initiation and development in Dianthus allwoodii and Dianthus alpinus. Sci. Hortic. 86, 57-70. 